\section{Structure of the Ionosphere}

IN a recent paper, Appleton and Builder ${ }^{1}$ mention a striking fact that on certain occasions, when the ionosphere is being surveyed by the 'pulse' method, there is a reversal in the times of arrival of the two magneto-ionic components into which the upgoing ray is split. They attribute this to differential group retardation, and it occurred to me a short time ago that this effect might throw some further light on a suggestion I have made previously ${ }^{2}$, namely, that the whole intermediate space between the $E$ and $F$ layers is ionised to a value very little less than that of the top of the $E$ layer.

I have made some approximate calculations of this group retardation, which indicate that a time delay sufficient to produce the effect noted by Appleton and Builder cannot be produced by a discrete layer $15-20 \mathrm{~km}$. thick without assuming that the density of such a layer can remain constant at a certain value for an appreciable time to a limit of about 1 part in 10,000. If, however, the whole of this intermediate space is ionised, the limit becomes of the order of 10 per cent.

This seems to suggest very strongly that the ionisation of the whole of the intermediate region is comparable with that of the top of the $E$ layer, and I hope to publish the results shortly; but, if this is the case, the group velocity throughout this region will be considerably less than its normal value in free space; and this may affect results derived from observations on the intermediate and $F$ layers. In fact, it raised the question as to whether the 'shelves' have a real existence or merely arise as points of inflexion on a group-velocity curve, and this requires further consideration.

Eilectrical Engineering Department,

\section{J. HOLLINGWORTH.}

College of Technology, Manchester.

Aug. 19.

1 Proc. Phys. Soc., 45, 208.

J. Inst. Elect. Eng., 72, No. 435, March 1933.

Chemical Separation of the Radioactive Element from its Bombarded Isotope in the Fermi Effect

Following the pioneer experiment of Fermi, it has been found by Fermi, Amaldi, D'Agostino, Rasetti and Segre that many elements up to the atomic number 30 , when bombarded by neutrons from a radon-beryllium source, are transmuted into a radioactive element which is chemically different from the bombarded element. In several cases of this type, they succeeded in separating chemically the active substance from the bulk of the bombarded element, and there is no inherent difficulty in getting any desirable concentration of the radioactive element.

They have not observed such chemical changes in elements above the atomic number 30, though many of these heavier elements show strong Fermi effects. For some of these, for example, arsenic, bromine, iodine, iridium and gold, they could show that the activity is carried by the bombarded element, which in the circumstances leads to the conclusion that the radioactive element is an isotope of the bombarded element.

In order to separate the radioactive isotope of the bombarded element from the bulk of the bombarded element, one has to find a new principle of separation. We have attempted to apply the following principle. If we irradiate by a neutron source a chemical compound of the element in which we are interested, we might expect those atoms of the element which are struck by a neutron to be removed from the compound. Whether the atoms freed in this way will interchange with their isotopes bound in the irradiated chemical compound will depend on the nature of the chemical compound with which we have to deal. If we work under conditions in which such an interchange does not take place, we obtain the radioactive isotope 'free', and by separating the 'free' element from the compound we can obtain any desirable concentration of the radioactive isotope.

We have applied this principle to iodine. Ethyl iodide has been irradiated and a trace of free iodine added to protect the radioactive isotope. By reduction and precipitation as silver iodide in water, it was easy to concentrate the activity so as to get from the precipitate ten times as many impulses of the GeigerMuller $\beta$-ray counter as directly from the irradiated ethyl iodide ${ }^{1}$. Apparently a large fraction of the active substance could be extracted from the ethyl iodide. The quantity of the active element obtainable in the precipitate will naturally depend on the quantity of the compound subjected to irradiation.

This principle of isotopic separation has also been applied to some other elements which, like iodine, are transmuted into their own isotopes, and further experiments mostly with organic compounds are in progress.

Physics Department,

LeO SzILARD, Medical College,

St. Bartholomew's Hospital, London, E.C.I. Sept. 10.

${ }^{1}$ Proc. Roy. Soc., A, 146, 483; 1934.

\section{Activated States in the Spectrum of Copper Hydride}

IN addition to the well-known band system ${ }^{1} \Sigma^{*} \rightarrow 1 \Sigma$ in copper hydride, we recently reported ${ }^{1}$ a new band system $1 \Sigma * * \rightarrow 1 \Sigma$, the activated states $1 \Sigma *$, ${ }^{1}{ }^{1} * *$ forming a doublet $\left(\nu_{e}^{*}=23431, \nu_{e}^{* *}=26369\right)$, pre viously known in the spectra of gold hydride $v_{e}^{*}=27658, v_{e}^{* *}=38231$ ). In the spectrum of silver hydride ${ }^{3},{ }^{1} \Sigma * *$ has not been found, although its presence is indicated by irregularities in $1{ }^{1} *$.

Extending our analysis into the ultra-violet region in the spectrum of copper hydride (in emission), we were able to disentangle two new band systems, composed of $P-Q \cdot R$-branches and forming $1 \Pi \rightarrow 1 \Sigma$ systems, the constants of the 1 II-terms being given below :

$$
\begin{aligned}
& \begin{array}{lccc} 
& \nu_{0} & B_{0} & \gamma_{0} \\
{ }^{1} \Pi^{*} & 27100 & 6,39 & 0,018 \\
{ }^{1} \Pi^{*} * & 27957 & 6,065 & 0,066
\end{array} \\
& \text { ( } \gamma_{0}=\Lambda \text {-doubling coefficient). }
\end{aligned}
$$

A more detailed account will appear later.

A. HEIMER.

T. HeIMER.

Laboratory of Physics,

University of Stockholm. Aug. 2.

1 A. Heimer and T. Heimer, Z, Phys., 84, 222; 1933.

2 E. Hulthén and R. V. Zumstein, Phys. Rev., 28, 13 ; 1926.

E. Bengtsson-Knave, Dissertation, Stockholm, 1932 . 